\title{
The Differential Transform
}

\section{Eng. Mahmoud Abu Hilal}

Science and Engineering, Al-Qasemi College, Baka, Israel

Email: mahmoudhilal@gmail.com

How to cite this paper: Hilal, E.M.A (2021) The Differential Transform. Journal of Applied Mathematics and Physics, 9, 1978-1992.

https://doi.org/10.4236/jamp.2021.98129

Received: June 25, 2021

Accepted: August 22, 2021

Published: August 25, 2021

Copyright (C) 2021 by author(s) and Scientific Research Publishing Inc. This work is licensed under the Creative Commons Attribution International License (CC BY 4.0).

http://creativecommons.org/licenses/by/4.0/

\begin{abstract}
One of the methods of mathematical analysis in many cases makes it possible to reduce the study of differential operators, pseudo-differential operators and certain types of integral operators and the solution of equations containing them, to an examination of simpler algebraic problems. The development and systematic use of operational calculus began with the work of O. Heaviside (1892), who proposed formal rules for dealing with the differentiation operator $\mathrm{d} / \mathrm{dt}$ and solved a number of applied problems. However, he did not give operational calculus a mathematical basis; this was done with the aid of the Laplace transform; J. Mikusiński (1953) put operational calculus into algebraic form, using the concept of a function ring [1]. Thereupon I'm suggesting here the use of the integration operator $\mathrm{dt}$ to make an extension for the systematic use of operational calculus. Throughout designing and analyzing a control system, we need to treat the functionality of the system with respect to time. The reaction of the system instructs us how to stable it by amplifiers and feedbacks [2], thereafter the Differential Transform is a good tool for doing this, and it's a technique to frustrate difficulties we may bump into, also it has the methods to find the immediate reaction of the system with respect to infinitesimal (tiny) time which spares us from the hard work in finding the time dependent function, this could be done by producing finite series.
\end{abstract}

\section{Keywords}

Operational Calculus, Time Domain, Differential Domain, Serieses, Difference to Differential Equation

\section{Introduction}

The Differential Transform shifts differential system from time plane to Cartesian plane that depends on the operator $\mathrm{dt}$, this new presentation makes it easier to be solved. The Differential Transform is very useful in solution of problems 
for linear ordinary differential equations [3], analyzing electric systems [4] and solving physical problems [5].

Here we define the Differential Transform as a theme and present several examples and calculate the Transform for the basic functions. It may be regarded as a nice exercise from the mathematical point of view, also it might have some applications to engineers.

In this article we'll attempt to interpret the Differential Transform by presenting its definition and properties. The examples accompanying, demonstrate the convenient usage of this transform and improve understanding its concept.

The uniqueness of this Transform refers to the direct inversion which gives the series of the functions. The inversion is easy and in case of a complicated function produces a finite series of the function which will be regarded as its abbreviation.

The main advantages of this Transform are in Digital Signal Processing (DSP) [6] where a difference equation of a filter could be treated the same as a differential equation.

\section{The Main Theorem}

Here is the essence of the Differential Transform presented by this definition:

Let the Differential Transform of $f_{(t)}$ be denoted as $d f_{(t)}$ and define it as

$$
d f_{(t)}=\frac{1}{d t} \int_{0}^{\infty} e^{\frac{-\tau}{d t}} f_{(\tau)} d \tau
$$

where the operator $d t$ represents the integrator $\int_{0}^{t} d t$.

\section{The Properties}

In our all next discussion we'll regard $G_{(d t)}=d f_{(t)}$ and $F_{(s)}$ the Laplace Transform [7] of $f_{(t)}$.

\subsection{Integrating}

For $f_{(t)}=\int_{0}^{t} f_{(t)}^{\prime} d t+f_{(0)}$ the Differential Transform is

$$
d f_{(t)}=d f_{(t)}^{\prime} d t+f_{(0)}
$$

\subsection{Differentiating}

The Differential Transform of $f_{(t)}^{\prime}=\frac{d}{d t} f_{(t)}$ is

$$
d f_{(t)}^{\prime}=\frac{d f_{(t)}-f_{(0)}}{d t}=\frac{\left.d f\right|_{0} ^{d t}}{d t}
$$

\subsection{Linearity}

Simply by switching $G_{(d t)}=d f_{(t)}$ from $t$ to the $c t, c=$ constant

$$
d f_{(c t)}=G_{\left(d_{c t}\right)}=G_{(c d t)}
$$




\subsection{Splitting}

For complex functions $G_{(d t)}=d f_{(t)}$ the $d(\operatorname{Real}(f))=\operatorname{Real}(G)$ and the

$$
d(\operatorname{Imaginary}(f))=\operatorname{Imaginary}(G)
$$

\subsection{Limiting}

$$
f_{(0)}=G_{(0)}
$$

\subsection{Shifting}

Having $G_{(d t)}=d f_{(t)}$ with $t \geq \tau$ we get $d f_{(t-\tau)}=G_{(d t)} e^{-\frac{\tau}{d t}}$.

\subsection{Convolution}

If $d h_{(t)}=d f_{(t)} d t d y_{(t)}$ then

$$
h_{(t)}=f(t) * y(t)=\int_{0}^{t} f(t-\tau) y(\tau) d \tau
$$

\subsection{Low Shaking}

$$
d\left(t^{n} \frac{d^{n}}{d t} f_{(t)}\right)=d_{t}^{n} \frac{d^{n}}{d d t} d f
$$

$\frac{d^{n}}{d d t} d f$ is the $n$-th derivation of $d f$ dependent to $d d t$.

\subsection{Inverse Low Shaking}

$$
d\left(\int_{0}^{t} \frac{\left.f_{(t)}\right|_{0} ^{t}}{t} d t\right)=\int_{0}^{d t} \frac{\left.d f\right|_{0} ^{d t}}{d t} d d t=\int_{0}^{d t} d f^{\prime} d d t
$$

$\int_{0}^{d t} d f^{\prime} d d t$ is the integral of $d f^{\prime}$ dependent to $d d t$.

\subsection{High Shaking}

$$
d\left(\frac{d^{n}}{d t} t^{n} f_{(t)}\right)=\frac{d^{n}}{d d t} d_{t}^{n} d f
$$

$\frac{d^{n}}{d d t} d_{t}^{n} d f$ is the $n$-th derivation of $d_{t}^{n} d f$ dependent to $d d t$.

\subsection{Inverse High Shaking}

$$
d\left(\frac{1}{t} \int_{0}^{t} f_{(t)} d t\right)=\frac{1}{d t} \int_{0}^{d t} d f d d t
$$

$\int_{0}^{d t} d f d d t$ is the integral of $d f$ dependent to $d d t$. 


\subsection{Particular Low Shaking}

$$
d\left(t^{n} f_{(t)}\right)=d_{t}^{n} \frac{d^{n}}{d d t}\left(d_{t}^{n} d f\right)
$$

$\frac{d^{n}}{d d t} d_{t}^{n} d f$ is the n-th derivation of $d_{t}^{n} d f$ dependent to $d d t$.

\section{Implementation Examples}

Now let's consider DT as an abbreviation for Differential Transform and make some DT'es using the properties:

1) Knowing that $t=\int_{0}^{t} d t$ leads to

$$
d(t)=d t
$$
2) Let $f_{(t)}=\frac{t^{2}}{2}, f_{(t)}^{\prime}=t$ following Equation (1) $\frac{t^{2}}{2}=\int_{0}^{t} t d t$ and its DT
would be:

$$
d\left(\frac{t^{2}}{2}\right)=d t d t=d_{t}^{2} \Rightarrow d\left(\frac{t^{2}}{2}\right)=d_{t}^{2}
$$
3) Let $f_{(t)}=\frac{t^{3}}{3 !}, f_{(t)}^{\prime}=\frac{t^{2}}{2 !}$ and following Equation (1) $\frac{t^{3}}{3 !}=\int_{0}^{t} \frac{t^{2}}{2 !} d t \quad$ and its
DT would be:

$$
d\left(\frac{t^{3}}{3 !}\right)=d\left(\frac{t^{2}}{2 !}\right) d t=d_{t}^{2} d t=d_{t}^{3} \Rightarrow d\left(\frac{t^{3}}{3 !}\right)=d_{t}^{3}
$$

4) Proceeding so on using Equation (1) we'll come $f_{(t)}=\frac{t^{n}}{n !}, \quad f_{(t)}^{\prime}=\frac{t^{n-1}}{(n-1) !}$ with $\frac{t^{3}}{3 !}=\int_{0}^{t} \frac{t^{2}}{2 !} d t$ its DT would be

$$
d\left(\frac{t^{n}}{n !}\right)=d\left(\frac{t^{n-1}}{(n-1) !}\right) d t=d_{t}^{n-1} d t=d_{t}^{n} \Rightarrow d\left(\frac{t^{n}}{n !}\right)=d_{t}^{n}
$$

5) Substituting Equation (12) in Equation (2) $f_{(t)}=t, f_{(t)}^{\prime}=1$ so $1=\frac{d}{d t} t$ its DT would be

$$
d(1)=\frac{d(t)-f(0)}{d t}=\frac{\left.d t\right|_{0} ^{t}}{d t}=\frac{d t-0}{d t}=1 \Rightarrow d(1)=1
$$

6) The DT of a constant $c$, using Equation (16) and (3) is

$$
d(c)=c d(1)=c \cdot 1=c \quad d(c)=c
$$

7) Now we can make $d\left(e^{t}\right), e^{t}=1+t+\frac{t^{2}}{2}+\frac{t^{3}}{3 !}+\cdots+\frac{t^{n}}{n !} \quad n \rightarrow \infty$ so

$d\left(e^{t}\right)=d(1)+d(t)+d\left(\frac{t^{2}}{2}\right)+d\left(\frac{t^{3}}{3 !}\right)+\cdots+d\left(\frac{t^{n}}{n !}\right) \quad \Rightarrow$

$d\left(e^{t}\right)=1+d_{t}+d_{t}^{2}+d_{t}^{3}+\cdots+d_{t}^{n}$

Here we have a finite series sum cause $d t$ is infinitesimal

$$
d\left(e^{t}\right)=\frac{1}{1-d t}
$$


8) The DT of $e^{i \omega t}=\cos (\omega t)+i \sin (\omega t)$ is $d e^{i \omega t}=d \cos (\omega t)+i d \sin (\omega t)$ and by applying

Linearity, Equation (3), on $d\left(e^{t}\right)=\frac{1}{1-d t}$ via replacing $t$ with $i \omega t$ gives:

$$
d e^{i \omega t}=\frac{1}{1-d_{i \omega t}}=\frac{1}{1-i \omega d_{t}}=\frac{1+i \omega d_{t}}{1+\omega^{2} d_{t}^{2}}=\frac{1}{1+\omega^{2} d_{t}^{2}}+i \frac{\omega d_{t}}{1+\omega^{2} d_{t}^{2}}
$$

now splitting (Equation (4)) gives $d \cos (\omega t)=\frac{1}{1+\omega^{2} d_{t}^{2}}$ and the imaginary part

$$
d \sin (\omega t)=\frac{\omega d t}{1+\omega^{2} d_{t}^{2}}
$$

9) Let's calculate the DT of $t e^{t}$ by shaking (Equation (11)):

$$
d\left(t e^{t}\right)=d t \frac{d}{d d t} d t d e^{t}=d t\left(\frac{d t}{1-d t}\right)^{\prime}=\frac{d t}{(1-d t)^{2}} \text {. }
$$

\section{The DT of Some Basic Function Is Shown in Table 1}

See Table 1.

\section{Solving Differential Equations}

\subsection{First Order Differential Equation}

Differential equations of the form $m f^{\prime}+n f=x$ with the initial $f(0)=a$.

$d f^{\prime}$ as to Equation (2) is $d f^{\prime}=\frac{d f-f(0)}{d t}=\frac{d f-a}{d t}=\frac{d f}{d t}-\frac{a}{d t}$ and the DT of $m f^{\prime}+n f=x$ is

$$
\begin{gathered}
m \frac{d f}{d t}-m \frac{a}{d t}+n d f=d x \\
d f(m+n d t)=d x d t+m a \Rightarrow \\
d f=\frac{d x d t+m a}{n d t+m}
\end{gathered}
$$

Example: solve $f^{\prime}+f=e^{-t}$ with the initial $f_{(0)}=0$.

We'll substitute $m=1, n=1, x=e^{-t}, a=0$ in Equation (20):

$$
d f=\frac{d e^{-t} d t+1 \cdot 0}{d t+1} \text { and since } d e^{-t}=\frac{1}{d t+1} \text { we obtain } d f=\frac{d t}{(d t+1)^{2}} .
$$

Now looking in Table 1 we'll found that $f_{(t)}=t e^{-t}$.

\subsection{Second Order Differential Equation}

Differential equations of the form $k f^{\prime \prime}+m f^{\prime}+n f=x$ with the initials

$$
\begin{aligned}
& f(0)=a, f^{\prime}(0)=b \quad d f^{\prime} \text { as to Equation (2) is } d f^{\prime}=\frac{d f-f(0)}{d t} \text { and } d f^{\prime \prime} \text { is } \\
& d f^{\prime \prime}=\frac{d f^{\prime}-f^{\prime}(0)}{d t}=\frac{d f-f(0)-f^{\prime}(0) d t}{d_{t}^{2}} \text {. }
\end{aligned}
$$


Table 1. The DT'es of the basic function.

\begin{tabular}{|c|c|c|c|}
\hline & The function & The Differential Transform & Where \\
\hline 1 & $f_{(t)}$ & $d f_{(t)}=\frac{1}{d t} \int_{0}^{\infty} e^{\frac{-\tau}{d t}} f_{(\tau)} d \tau$ & \\
\hline 2 & $h(t)=f(t) * y(t)=\int^{t} f_{(t-\tau)} y_{(\tau)} d \tau$ & $d h_{(t)}=d f_{(t)} d t d y_{(t)}$ & \\
\hline 3 & $f_{(t-\tau)}, \quad t \geq \tau$ & $e^{-\frac{\tau}{d t}} d f_{(t)}$ & \\
\hline 4 & $e^{-a t} f(t)$ & $\frac{1}{1+d a t} G\left(\frac{d t}{1+d a t}\right), \quad G=d f$ & \\
\hline 5 & unit impulse $\delta_{(t)}$ & $1 / d t$ & \\
\hline 6 & $t^{n} / n !$ & $d_{t}^{n}$ & \\
\hline 7 & Constant: $C$ & C & \\
\hline 8 & $e^{-\alpha t}$ & $1 /(1+d \alpha t)$ & \\
\hline 9 & $\sin (\omega t)$ & $d \omega t /\left(1+d_{\omega t}^{2}\right)$ & \\
\hline 10 & $\cos (\omega t)$ & $1 /\left(1+d_{\omega t}^{2}\right)$ & \\
\hline 11 & $\sinh (\omega t)$ & $d \omega t /\left(1-d_{\omega t}^{2}\right)$ & \\
\hline 12 & $t \sinh \omega t$ & $\frac{2 d_{\omega t}^{2}}{\omega\left(1-d_{\omega t}^{2}\right)^{2}}$ & \\
\hline 13 & $\cosh (\omega t)$ & $1 /\left(1-d_{\omega t}^{2}\right)$ & \\
\hline 14 & $t \cosh \omega t$ & $d t \frac{1+d_{\omega t}^{2}}{\left(1-d_{\omega t}^{2}\right)^{2}}$ & \\
\hline 15 & $t^{n} e^{-\alpha t}$ & $d_{t}^{n} /(1+\alpha d t)^{n+1}$ & \\
\hline 16 & $t \sin \omega t$ & $\frac{2 d_{\omega t}^{2}}{\omega\left(1+d_{\omega t}^{2}\right)^{2}}$ & \\
\hline 17 & $\sin \omega t / t$ & $\arctan (d \omega t / d t)$ & \\
\hline 18 & $t \cos \omega t$ & $d t \frac{1-d_{\omega t}^{2}}{\left(1+d_{\omega t}^{2}\right)^{2}}$ & \\
\hline 19 & $\frac{1-\cos \omega t}{t}$ & $\frac{\ln \sqrt{1+d_{\omega t}^{2}}}{d t}$ & \\
\hline 20 & $e^{-\alpha t} \sin \omega t$ & $\frac{\omega d t}{d_{\omega_{n} t}^{2}+2 \alpha d t+1}$ & $\alpha+i \omega=\omega_{n} e^{i \theta}$ \\
\hline 21 & $e^{-\alpha t} \cos \omega t$ & $\frac{1+\alpha d t}{d_{\omega_{n} t}^{2}+2 \alpha d t+1}$ & $\omega_{n}=\sqrt{\alpha^{2}+\omega^{2}}$ \\
\hline 22 & $\frac{e^{-\alpha t}}{\sin \theta} \sin (\theta-\omega t)$ & $\frac{1}{d_{\omega_{n} t}^{2}+2 \alpha d t+1}$ & $\theta=\arctan (\omega / \alpha)$ \\
\hline 23 & $1-\frac{e^{-\alpha t}}{\sin \theta} \sin (\omega t+\theta)$ & $\frac{d_{\omega_{n} t}^{2}}{d_{\omega_{n} t}^{2}+2 \alpha d \omega_{n} t+1}$ & $\alpha=\omega_{n} \cos \theta$ \\
\hline 24 & $\omega_{n} e^{-\alpha t} \cos (\omega t-\theta)$ & $\frac{\omega_{n}^{2} d t+\alpha}{d_{\omega_{n} t}^{2}+2 \alpha d t+1}$ & $\omega=\omega_{n} \sin (\theta)$ \\
\hline
\end{tabular}


The DT of $k f^{\prime \prime}+m f^{\prime}+n f=x$ becomes

$$
\begin{gathered}
k \frac{d f}{d_{t}^{2}}-k \frac{a}{d_{t}^{2}}-k \frac{b}{d t}+m \frac{d f}{d t}-m \frac{a}{d t}+n d f=d x \\
d f\left(k+m d t+n d_{t}^{2}\right)=d x d_{t}^{2}+k a+k b d t+m a d t \\
d f=\frac{d x d_{t}^{2}+(m a+k b) d t+k a}{n d_{t}^{2}+m d t+k}
\end{gathered}
$$

Example: solve $f^{\prime \prime}+f=0$ with the initials $f(0)=4, f^{\prime}(0)=-1$.

We'll substitute $k=1, m=0, n=1, x=0, a=4, b=-1$ in Equation (21):

$$
d f=\frac{0+(0-1) d t+4}{d_{t}^{2}+1}=\frac{4}{1+d_{t}^{2}}-\frac{d t}{1+d_{t}^{2}}
$$

Now looking in Table 1 we'll found that

$$
f_{(t)}=4 \cos (t)-\sin (t) \text {. }
$$

\section{Electrical Circuits}

Here we transferred the electrical circuit elements to the differential domain as shown in Figure 1.

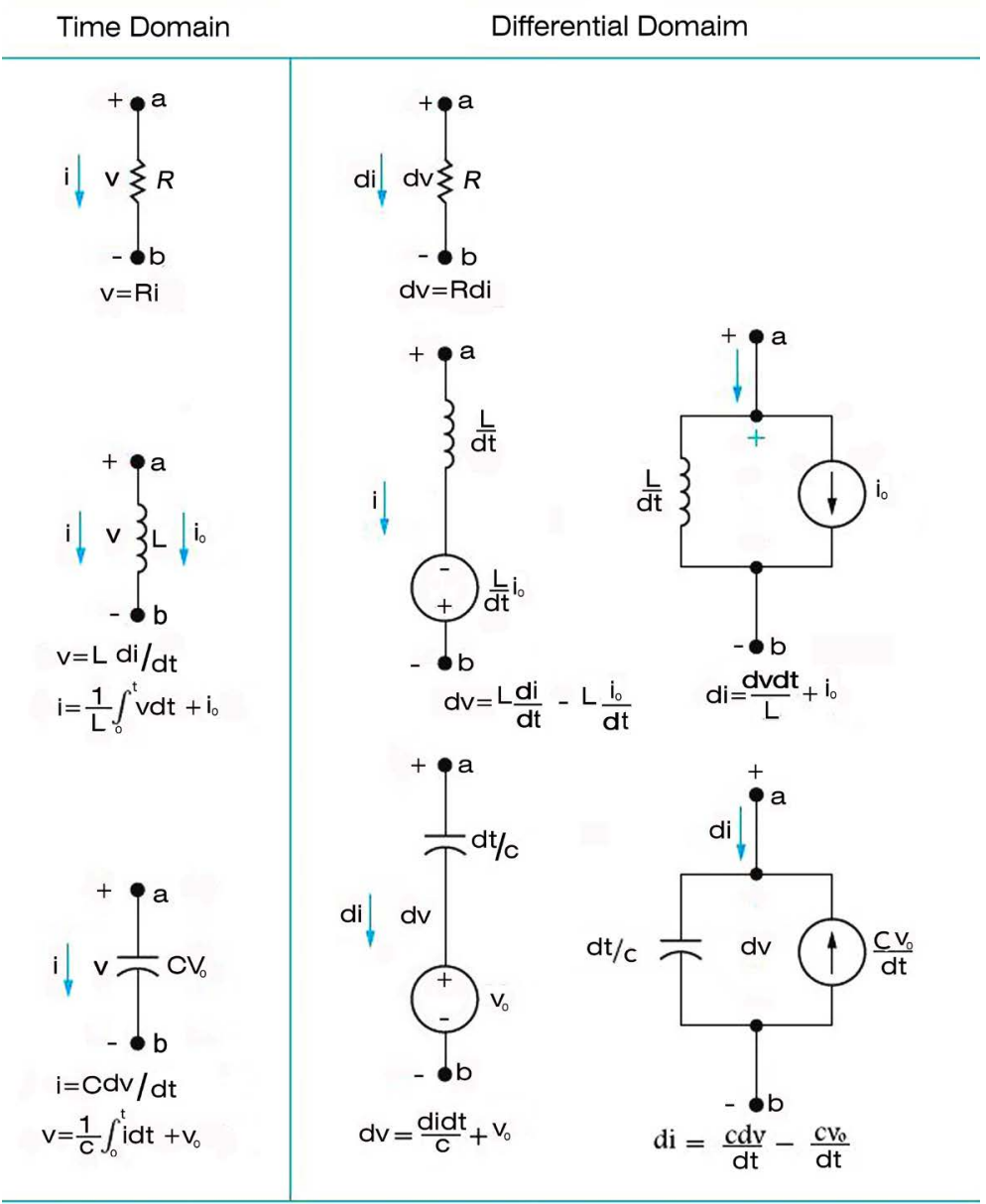

Figure 1. The transformed circuits the differential domain. 


\subsection{RC Circuit}

The transformed circuit in Figure 2 is shown in Figure 3.

The circuit is fed with $v(t)=v$ DC volts and we need to find $i(t)$ using Kirchoff's law:

$$
\begin{gathered}
v(t)=R i(t)+\frac{1}{c}\left(\int_{0}^{t} i(t) d t+q_{0}\right) \text { so } d v=R d i+\frac{1}{c} d i d t+\frac{q_{0}}{c} \\
d i=\frac{v-\frac{q_{0}}{c}}{R+\frac{d t}{c}}=\frac{v-\frac{q_{0}}{c}}{R} \cdot \frac{1}{1+d \frac{t}{R C}} \Rightarrow i(t)=\frac{v-\frac{q_{0}}{C}}{R} e^{-\frac{t}{R C}}
\end{gathered}
$$

\subsection{RL Circuit}

Applying the above steps to the circuit in shown in Figure 4:

$$
\begin{aligned}
& v(t)=R i(t)+L \frac{d}{d t} i(t) \Rightarrow \text { the DT is } d v=v=R d i+L \frac{d i-i_{0}}{d t}=R d i+L \frac{d i}{d t}-L \frac{i_{0}}{d t} \\
& d i=\frac{v+L i_{0} / d t}{R+L / d}=\frac{v}{R+L / d t}+\frac{L i_{0} / d t}{R+L / d t}=\frac{v}{R} \cdot \frac{d \frac{R}{L} t}{1+d \frac{R}{L} t}+i_{0} \frac{1}{1+d \frac{R}{L} t}
\end{aligned}
$$

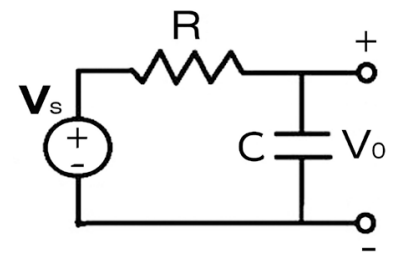

Figure 2. RC circuit.

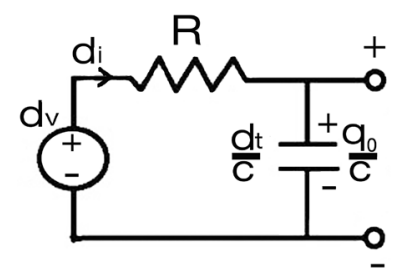

Figure 3. The DT of the circuit in Figure 2.

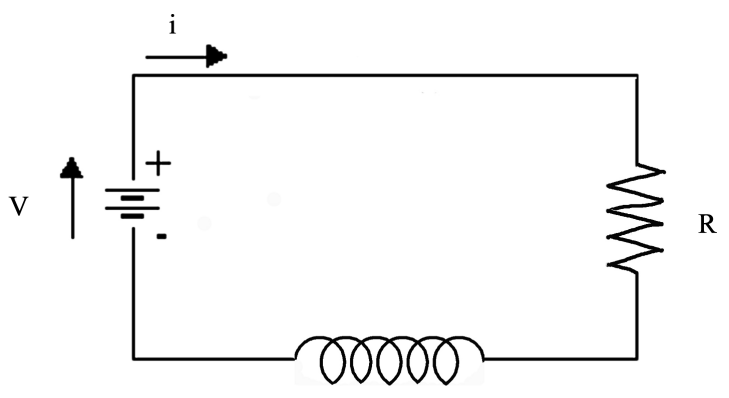

$\mathrm{L}$

Figure 4. RL circuit. 


$$
\begin{gathered}
i(t)=\frac{v}{R}\left(1-e^{-\frac{R}{L} t}\right)+i_{0} e^{-\frac{R}{L} t}=\frac{v}{R}+\left(i_{0}-\frac{v}{R}\right) e^{-\frac{R}{L} t} \\
i(t)=\frac{v}{R}+\left(i_{0}-\frac{v}{R}\right) e^{-\frac{R}{L} t} .
\end{gathered}
$$

\section{Physical Problems}

\section{Harmonic Motion}

The differential equation of the physics harmonic motion discribed in Figure 5.

$$
m a+k x=0
$$

where the $m$ is the mass, $a=x^{\prime \prime}$ is the acceleration, $k$ is the spring constant, $A=x_{0}$ is the amplitude with the initials $x(0)=A, x^{\prime}(0)=v_{0}=0$.

The DT of $m d x^{\prime \prime}+k d x=0$ where $d x^{\prime \prime}=\frac{d x-x_{0}}{d_{t}^{2}}=\frac{d x}{d_{t}^{2}}-\frac{A}{d_{t}^{2}}$ is

$m \frac{d x}{d_{t}^{2}}-m \frac{A}{d_{t}^{2}}+k d x=0 \Rightarrow m d x-m A+k d x d_{t}^{2}=0$ giving $d x=\frac{A}{1+\frac{k}{m} d_{t}^{2}}$ and by Linearity (Equation (3)) $d x=\frac{A}{1+\frac{k}{m} d_{t}^{2}}=\frac{A}{1+d_{t \sqrt{\frac{k}{m}}}^{2}} \quad x(t)=A \cos \left(t \sqrt{\frac{k}{m}}\right)$.

\section{Conversion between Laplace Transform (LT) [7] and Differential Transform (DT)}

$$
G=\frac{1}{d t} F_{\left(\frac{1}{d t}\right)} \text { and } F=\frac{1}{s} G_{\left(\frac{1}{s}\right)}
$$

1) Let's convert the DT of $f_{(t)}=\sin (t)$ to LT:

$$
G=d f=d \sin t=\frac{d t}{1+d_{t}^{2}} \text { therefore } F=\frac{1}{s} G_{\left(\frac{1}{s}\right)}=\frac{1}{s} \frac{\frac{1}{s}}{1+\left(\frac{1}{s}\right)^{2}}=\frac{1}{1+s^{2}}
$$

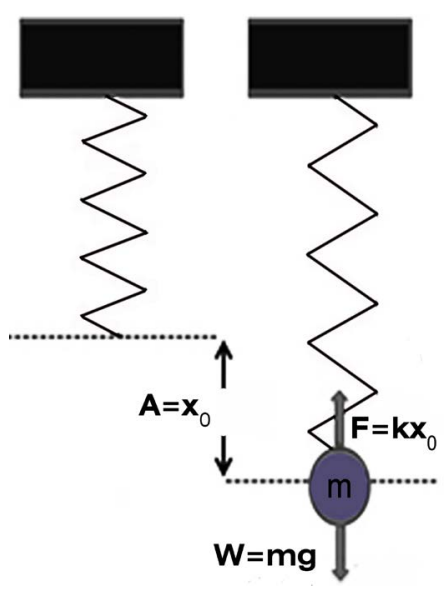

Figure 5. Harmonic motion. 
2) Let's convert the LT of $f_{(t)}=e^{-t}$ to DT:

$$
F_{(s)}=\frac{1}{s+1} \text { so } G=\frac{1}{d t} F_{\left(\frac{1}{d t}\right)}=\frac{1}{d t} \frac{1}{\frac{1}{d t}+1}=\frac{1}{1+d t}
$$

\section{The DT Inversion}

The inversion of $d f_{(t)}$ is $f_{(t)}$, it means that we transfer the function from the differential domain to the time domain.

1) Inversion via Convolution (Equation (6)).

Example: Find $f(t)$ if $d f=\frac{d t}{-12 d_{t}^{2}-d t+1}$.

$$
\begin{aligned}
d f & =\frac{d t}{-12 d_{t}^{2}-d t+1}=\frac{d t}{(1+3 d t)(1-4 d t)}=\frac{1}{(1+d 3 t)} d t \frac{1}{(1-d 4 t)} \\
& =d e^{-3 t} d t d e^{4 t}=d\left(e^{-3 t} * e^{4 t}\right)
\end{aligned}
$$

see Table 2.

\begin{tabular}{|c|c|c|}
\hline & The function & The series \\
\hline 1 & $f_{(t)}$ & $A_{n}$ \\
\hline 2 & $\alpha e^{t}$ & $\alpha$ \\
\hline 3 & $t e^{t}$ & $n$ \\
\hline 4 & $\alpha e^{\beta t}$ & $\alpha \beta^{n}$ \\
\hline 5 & $t e^{\alpha t}$ & $n \alpha^{n}$ \\
\hline 6 & $\sin \left(\omega_{0} t\right)$ & $\omega_{0}^{n} \sin \left(n \frac{\pi}{2}\right)$ \\
\hline 7 & $\cos \left(\omega_{0} t\right)$ & $\omega_{0}^{n} \cos \left(n \frac{\pi}{2}\right)$ \\
\hline 8 & $\int_{0}^{1} f_{(t)} d_{t}$ & $A_{n-1}, \quad n-1 \geq 0$ \\
\hline 9 & $\int_{0}^{t} \cdots \int_{0}^{m} f_{(t)} d_{t} \cdots d_{t}, \mathrm{~m}$ times integration & $A_{n-m}, \quad n-m \geq 0$ \\
\hline 10 & $\frac{1}{t} \int_{0}^{t} f_{(t)} d_{t}$ & $\frac{1}{n+1} A_{n}$ \\
\hline 11 & $\int_{0}^{t}\left(f_{(t)} / t\right) d t$ & $\frac{1}{n} A_{n}$ \\
\hline 12 & $t^{m} \frac{d^{m}}{d_{t}^{m}} f(t), m$ times derivation & $\frac{n !}{(n-m) !} A_{n}$ \\
\hline
\end{tabular}

$$
\begin{aligned}
f_{(t)}=e^{-3 t} * e^{4 t}=\int_{0}^{t} e^{-3 \tau} e^{4(t-\tau)} d \tau & =e^{4 t} \int_{0}^{t} e^{-7 \tau} d \tau=\left.e^{4 t} \frac{e^{-7 \tau}}{-7}\right|_{0} ^{t}=\frac{1}{7} e^{4 t}-\frac{1}{7} e^{-3 t} \\
f_{(t)} & =\frac{1}{7} e^{4 t}-\frac{1}{7} e^{-3 t} .
\end{aligned}
$$

Table 2. Series of some basic functions. 


\section{2) The direct inversion}

We know from algebra that the sum of the series $r(x)=\sum_{k=0}^{\infty} x^{k}=1+x+x^{2}+x^{3}+\cdots$ for $|x|<1$ is $\operatorname{sum}(r(x))=\frac{1}{1-x}$, and will regard this formula as "The sum formula".

Hence $d(\sin t)=\frac{d t}{1+d_{t}^{2}}$ the sum formula is $\frac{1}{1+d_{t}^{2}}=1-d_{t}^{2}+d_{t}^{4}-d_{t}^{6}+\cdots$ and $d \sin t=\frac{d t}{1+d_{t}^{2}}=d t\left(1-d_{t}^{2}+d_{t}^{4}-\cdots\right)=d t-d_{t}^{3}+d_{t}^{5}-\cdots$ now inverting this equation gives $\sin t=1-\frac{t^{2}}{2 !}+\frac{t^{4}}{4 !}-\frac{t^{6}}{6 !}+\cdots$.

2) A good exercise is to invert $d f(t)=\frac{1}{1-d_{t}^{24}}$ according to the sum formula $d f(t)=\frac{1}{1-d_{t}^{24}}=1+d_{t}^{24}+d_{t}^{48}+\cdots$, and the series of $f(t)$ becomes $f(t)=1+\frac{t^{24}}{24 !}+\frac{t^{48}}{48 !}+\cdots$.

Notice that dealing with the series of $f_{(t)}$ is easier than dealing directly with $f_{(t)}$ specially when we are working with values of $t$ near the zero.

Means that the function behaves like $f(t)_{t \rightarrow 0} \approx 1+\frac{t^{24}}{24 !}+\frac{t^{48}}{48 !}$ and under these conditions the finite series of the function could be regarded as its abbreviation and we can be satisfied with $f(t)^{*}=1+\frac{t^{24}}{24 !}+\frac{t^{48}}{48 !}$.

3) A function of variable approaching the zero i.e. the infinitesimal function of $f(t)$ is $f^{*}(t)=\left.f(t)\right|_{t \rightarrow 0}$.

Recall that: $g(t)=\sin t=t-\frac{t^{3}}{3 !}+\frac{t^{5}}{5 !}-\frac{t^{7}}{7 !}+\cdots \Rightarrow g^{*}(t)=\left.\sin t\right|_{t \rightarrow 0}=t$.

Example: To find $f^{*}(t)$ where $d f(t)=\frac{(1+a d t)^{n}}{(1+b d t)^{m}}$ and $t \rightarrow 0$ we can replace $(1+a d t)^{n}$ with $1+$ andt and $\frac{1}{(1+b d t)^{m}}$ with 1 -amdt and get $d f^{*}(t)=(1+a n d t)(1-b m d t)=1+(a n-b m) d t-a b n m d_{t}^{2}$, $f^{*}(t)=1+(a n-b m) t-0.5 a b n m t^{2}$.

\section{Solving Differential Equations via Series Method}

\subsection{Taylor Series Form [8]}

$$
\begin{aligned}
f(x)= & f(a)+f^{\prime}(a)(x-a)+\frac{f^{\prime \prime}(a)}{2 !}(x-a)^{2}+\frac{f^{\prime \prime \prime}(a)}{3 !}(x-a)^{3}+\cdots \\
& +\frac{f^{(n)}(a)}{n !}(x-a)^{n}+\cdots
\end{aligned}
$$

And for $a=0$ :

$$
f(x)=f(0)+f^{\prime}(0) x+f^{\prime \prime}(0) \frac{x^{2}}{2 !}+f^{\prime \prime \prime}(0) \frac{x^{3}}{3 !}+f^{\prime \prime \prime \prime}(0) \frac{x^{4}}{4 !}+\cdots
$$


The DT of Tylor series:

$$
\begin{gathered}
d f=f(0)+f^{\prime}(0) d x+f^{\prime \prime}(0) d_{x}^{2}+f^{\prime \prime \prime}(0) d_{x}^{3}+f^{\prime \prime \prime \prime}(0) d_{x}^{4}+\cdots \\
d f=\sum_{n=0}^{\infty} K_{n} d_{t}^{(n)}
\end{gathered}
$$

where the series

$$
K_{n}=\left\{f(0), f^{\prime}(0), f^{\prime \prime}(0), f^{\prime \prime \prime}(0), f^{\prime \prime \prime \prime}(0), \cdots, f^{(n)}(0)\right\}
$$

\subsection{The Differentiation of $f$ Derived from the DT of Taylor Series}

$$
\begin{gathered}
d f^{\prime}=\frac{d f-f(0)}{d x}=f^{\prime}(0)+f^{\prime \prime}(0) d_{x}+f^{\prime \prime \prime}(0) d_{x}^{2}+f^{\prime \prime \prime \prime}(0) d_{x}^{3}+\cdots=A_{n} d_{t}^{(n)} \\
A_{n}=\left\{f^{\prime}(0), f^{\prime \prime}(0), f^{\prime \prime \prime}(0), f^{\prime \prime \prime \prime}(0), \cdots, f^{(n)}(0)\right\} \\
d f^{\prime \prime}=\frac{d f-f(0)-f^{\prime}(0) d x}{d_{x}^{2}}=f^{\prime \prime}(0)+f^{\prime \prime \prime}(0) d_{x}+f^{\prime \prime \prime \prime}(0) d_{x}^{2}+\cdots=B_{n} d_{t}^{(n)} \\
B_{n}=\left\{f^{\prime \prime}(0), f^{\prime \prime \prime}(0), f^{\prime \prime \prime \prime}(0), \cdots, f^{(n)}(0)\right\} \\
d f^{\prime \prime \prime}=\frac{d f-f(0)-f^{\prime}(0) d x-f^{\prime \prime}(0) d_{x}^{2}}{d_{x}^{3}} \\
=f^{\prime \prime \prime}(0)+f^{\prime \prime \prime \prime}(0) d_{x}+f^{(5)}(0) d_{x}^{2} \cdots=C_{n} d_{t}^{(n)} \\
C_{n}=\left\{f^{\prime \prime \prime}(0), f^{\prime \prime \prime \prime}(0), f^{(5)}(0), \cdots, f^{(n)}(0)\right\}
\end{gathered}
$$

The general form of linear differential equations order $m$ is $A_{m} f_{(t)}^{(m)}=x(t)$ with the initial conditions

$$
K_{m}=\left\{f(0), f^{\prime}(0), f^{\prime \prime}(0), f^{\prime \prime \prime}(0), f^{\prime \prime \prime \prime}(0), \cdots, f^{(m-1)}(0)\right\}
$$

And $x(t)=\sum_{n=0}^{\infty} B_{n} \frac{t^{n}}{n !}$. So we can perform the DT for the equation:

$$
\begin{aligned}
& A_{0} d f+A_{1}\left[\frac{d f}{d_{t}}-\frac{K_{0}}{d_{t}}\right]+A_{2}\left[\frac{d f}{d_{t}^{2}}-\frac{K_{0}}{d_{t}^{2}}-\frac{K_{1}}{d_{t}}\right]+A_{3}\left[\frac{d f}{d_{t}^{3}}-\frac{K_{0}}{d_{t}^{3}}-\frac{K_{1}}{d_{t}^{2}}-\frac{K_{2}}{d_{t}}\right] \\
& +\cdots+A_{m}\left[\frac{d f}{d_{t}^{m}}-\frac{K_{0}}{d_{t}^{m}}-\frac{K_{1}}{d_{t}^{m-1}}-\cdots-\frac{K_{m-1}}{d_{t}}\right]=d_{t}^{m} d x
\end{aligned}
$$

In series method solution the initials series for $n>m-1 \quad K_{n}=0$ so it doesn't effect finding.

The solution $f_{(t)}=\sum_{n=0}^{\infty} A_{n} \frac{t^{n}}{n !} \quad n=0,1,2, \cdots, \infty$ that we have $A_{n}=\left\{f(0), f^{\prime}(0), f^{\prime \prime}(0), f^{\prime \prime \prime}(0), f^{\prime \prime \prime \prime}(0), \cdots, f^{(m-1)}(0), \cdots\right\}$ and will start finding $A_{n}$ for $n \geq m$ so the relevant DT donated RTD is:

$$
A_{0} d_{t}^{m} d f+A_{1} d_{t}^{m-1} d f+A_{2} d_{t}^{m-2} d f+A_{3} d_{t}^{m-3} d f+\cdots+A_{m} d f=d_{t}^{m} d x
$$

\subsection{Deriving Series from the DT of Taylor Series}

Having 


$$
\begin{gathered}
d f=\sum_{n=0}^{\infty} K_{n} d_{t}^{(n)}, \\
K_{n}=\left\{f(0), f^{\prime}(0), f^{\prime \prime}(0), f^{\prime \prime \prime}(0), f^{\prime \prime \prime \prime}(0), \cdots, f^{(n)}(0)\right\} \\
G=d_{t}^{m} d f=\sum_{n=m}^{\infty} C_{n-m} d_{t}^{(n)}
\end{gathered}
$$

where $C_{n-m}=0$ for $(n-m)<0$.

And recalling (Equation (7)) $H=d\left(t^{m} \frac{d^{m}}{d t} f_{(t)}\right)=d_{t}^{m} \frac{d^{m}}{d d t} d f$.
Gives

$$
G=\sum_{n=m}^{\infty} \frac{n !}{(n-m) !} K_{n} d_{t}^{(n)}
$$

Example 11.3.1: solve $f^{\prime \prime}+f=0$ with the initials $f(0)=4, f^{\prime}(0)=-1$ $d f^{\prime \prime}+d f=0$.

The solution would be of the form $f_{(t)}=\sum_{n=0}^{\infty} A_{n} \frac{t^{n}}{n !}$ so we should find $A_{n}=$ ?.
1) Regular method:

$$
\begin{aligned}
\frac{d f-f(0)-f^{\prime}(0) d_{t}}{d_{t}^{2}}+d f=0 & \rightarrow d f-f(0)-f^{\prime}(0) d_{t}+d_{t}^{2} d f=0 \\
d f\left(1+d_{t}^{2}\right)=f(0)+f^{\prime}(0) d_{t} & \rightarrow d f=\frac{f(0)+f^{\prime}(0) d_{t}}{1+d_{t}^{2}} \\
& \rightarrow d f=\frac{4-d_{t}}{1+d_{t}^{2}} \ldots \text { (soll) }
\end{aligned}
$$

And the DT inversion of $d f \quad f(x)=4 \cos (t)-\sin (t)$.

2) Seriese method: the relevant transform RTD of the differential equation is $\rightarrow d_{t}^{2} d f+d f=0$.

And as to Equation (24). $A_{n} d_{t}^{n}+A_{n-2} d_{t}^{n}=0 \rightarrow A_{n}=-A_{n-2}$.

The initials gives $A_{0}=4, A_{1}=-1 \rightarrow A_{2}=-A_{0}=-4, A_{3}=-A_{1}=1$.

Example 11.3.2: Solve $t^{2} f^{\prime \prime}+f=e^{t}$.

We recall that $e^{t}=\sum_{n=0}^{\infty} \frac{t^{n}}{n !} \rightarrow d e^{t}=\sum_{n=0}^{\infty} d_{t}^{n}=\sum_{n=0}^{\infty} C_{n} d_{t}^{n}$ for $C_{n}=1$.

The solution would be of the form $f_{(t)}=\sum_{n=0}^{\infty} A_{n} \frac{t^{n}}{n !}$ and we should find $A_{n}=$ ? .

According to Equation (25): $\frac{n !}{(n-2) !} A_{n}+A_{n}=C_{n}=1$ so $A_{n}=\frac{1}{n^{2}-n+1}$,

$A_{0}=\frac{1}{0^{2}-0+1}=1, \quad A_{1}=\frac{1}{1^{2}-1+1}=1, \quad A_{2}=\frac{1}{2^{2}-2+1}=\frac{1}{3}, \quad A_{3}=\frac{1}{3^{2}-3+1}=\frac{1}{7}$,

Therefore we find $f(t)=\sum_{n=0}^{\infty} \frac{1}{n^{2}-n+1} * \frac{t^{n}}{n !}$.

\subsection{Table 2 Gives the Series of Some Basic Functions}

$$
\text { Concerning That } f_{(t)}=\sum_{n=0}^{\infty} A_{n} \frac{t^{n}}{n !}
$$

See Table 2. 


\section{A New Interpretation for Z Transform}

We can shift between the $\mathrm{Z}$ transform [9] and differential transform by substituting $d_{t}=Z^{-1}$.

Hence we resolve a difference equation [10] of digital filter via $Z$ transform and getting the solution as a series $y_{n}$. Means that the difference equation isn't other than a differential equation of a physical filter and it's solution is $f_{(t)}=\sum_{n=0}^{\infty} y_{n} \frac{t^{n}}{n !}$.

Example: Consider the difference equation $y_{n+1}-2 y_{n}=7$ with the initial $y_{0}=3$.

Applying $Z$ transform $z Y_{(z)}-3 z-2 Y_{(z)}=\frac{7 z}{z-1} \rightarrow Y_{(z)}=\frac{10 z}{z-2}-\frac{7 z}{z-1}$ gives $y_{n}=10 * 2^{n}-7$.

Actually this is the solution of the differential equation $f^{\prime}-2 f=7$ which is:

$$
f_{(t)}=\sum_{n=0}^{\infty} y_{n} \frac{t^{n}}{n !}=\sum_{n=0}^{\infty}\left(10 * 2^{n}-7\right) \frac{t^{n}}{n !}
$$

and from Table 2: $f_{(t)}=10 e^{2 t}-7 e^{t}$.

\section{Finalization}

We can learn from this paper that the Differential Transform is not a replacement for the existing methods, it could be useful in treating differential problems and for deriving the series of the solution. Here we found that difference equation which represents a digital filter could be treated the same as a differential equation of a physical filter. And a high potential is still embodied in this Transform.

\section{Conflicts of Interest}

The author declares no conflicts of interest regarding the publication of this paper.

\section{References}

[1] Krabbe (1970) Operational Calculus. First Edition, Springer-Verlag, Berlin/Heidelberg/New York.

[2] Doyle, J.C., Francis, B.A. and Tannenbaum, A.R. (2013) Feedback Control Theory, 3 Basic Concepts. Courier Corporation, North Chelmsford, Massachusetts.

[3] Rawat, K.S. (2007) Differential Equations. Garhwal Univarcity Campus Badshahithaul, Sarup \& Sons, New Delhi.

[4] Nilsson, J.W. and Riedel, S.A. (2008) Electric Circuits. Prentice Hall, Hoboken.

[5] Roche, J. (2002) Introducing Simple Harmonic Motion. Physics Education, 37, 497.

[6] Smith III, J.O. (2007) Introduction to Digital Filters with Audio Applications: Center for Computer Research in Music and Acoustics (CCRMA). Stanford University, Stanford, California.

[7] Hsu, R. (2018) Introduction to the Laplace Transform and Application. John Wiley \& Sons, Hoboken.

[8] Masjed-Jamei, M., Moalemi, Z., Area, I. et al. (2018) A New Type of Taylor Series 
Expansion. Journal of Inequalities and Applications, 2018, Article No. 116.

[9] Poularikas, A.D. (2000) The Z-Transform. CRC Press LLC, Boca Raton.

[10] Elaydi, S. (2005) An Introduction to Difference Equation. 3rd Edition, Department of Mathematics Trinity University San Antonio, Springer Science + Business Media, Inc., Berlin. 